STRUCTURAL BIOLOGY

ISSN 2059-7983

\title{
Facilitating best practices in collecting anomalous scattering data for de novo structure solution at the ESRF Structural Biology Beamlines
}

\author{
Daniele de Sanctis, ${ }^{*}$ Marcus Oscarsson, Alexander Popov, Olof Svensson and \\ Gordon Leonard
}

ESRF - The European Synchrotron, 71 Avenue des Martyrs, 38000 Grenoble, France. *Correspondence e-mail: desancti@esrf.fr

Received 24 July 2015

Accepted 18 January 2016

Keywords: ESRF; MAD; experimental phasing; MXCUBE2.

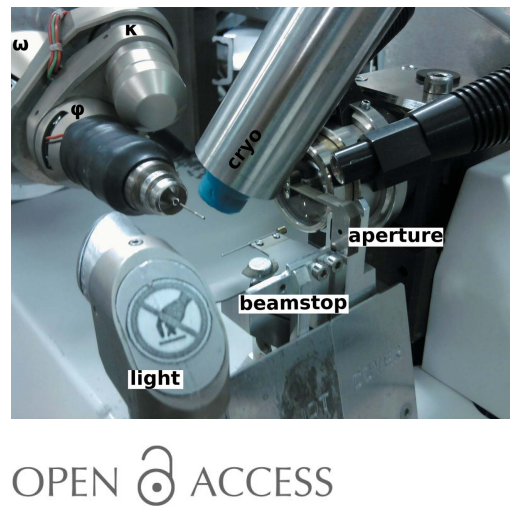

The constant evolution of synchrotron structural biology beamlines, the viability of screening protein crystals for a wide range of heavy-atom derivatives, the advent of efficient protein labelling and the availability of automatic dataprocessing and structure-solution pipelines have combined to make de novo structure solution in macromolecular crystallography a less arduous task. Nevertheless, the collection of diffraction data of sufficient quality for experimental phasing is still a difficult and crucial step. Here, some examples of good data-collection practice for projects requiring experimental phasing are presented and recent developments at the ESRF Structural Biology beamlines that have facilitated these are illustrated.

\section{Introduction}

The constantly increasing number of macromolecular crystal structures deposited in the Protein Data Bank (PDB; Berman et al., 2007), the increasing number of protein folds that these contain and the advent of more sensitive methods (McCoy et al., 2007; Read, 2001; DiMaio et al., 2011) has meant that molecular replacement (MR) is the overwhelming choice for structure solution in macromolecular crystallography (MX). However, the solution of the crystal structures of biological macromolecules of unknown fold and of large protein-protein or protein-nucleic acid complexes still often requires experimental phase determination. Most experiments for de novo structure determination routinely exploit anomalous scattering via the multiwavelength anomalous dispersion (MAD; Smith, 1991; Hendrickson, 1991) or single-wavelength anomalous dispersion (SAD; Rice et al., 2000; Dauter, 2002; Dauter et al., 2002) techniques. Such experiments are facilitated by the almost continuous evolution of tunable synchrotron beamlines at which experimenters are able to accurately measure the absorption edges of almost any anomalous scatterer that can be introduced into a crystal and to collect diffraction data at various energies around these in order to optimize anomalous and dispersive signals.

When carried out on a single cryocooled crystal, MAD and SAD experiments, in principle overcome the problems of non-isomorphism that can plague isomorphous replacement experiments. Nevertheless, in experiments where anomalous signals are small (i.e. S-SAD; Hendrickson \& Teeter, 1981; Dauter et al., 1999; Liu \& Hendrickson, 2015; Weinert et al., 2015) care must be taken to reduce systematic errors and, most of all, errors introduced by radiation damage. Radiation 
damage is exacerbated in MAD/SAD experiments because it not only results in a decrease, as a function of absorbed X-ray dose (Seltzer, 1993; Holton, 2009), in the resolution to which a crystal diffracts, it also causes specific chemical damage including disulfide-bond breakage (Weik et al., 2000; Leiros et al., 2001; Ennifar et al., 2002), changes in electronic state (Berglund et al., 2002; Schlichting et al., 2000) and, very importantly, reduction in the 'occupancy' of anomalous scatterers (Ramagopal et al., 2005; Evans et al., 2003; Ravelli et al., 2005). Moreover, specific radiation damage of the types mentioned above may already occur at doses much lower than the Garman Limit (Owen et al., 2006). Sometimes the effects of radiation damage can be used to an experimenter's advantage, as in radiation-damage-induced phasing (RIP; Ravelli et al., 2003; de Sanctis \& Nanao, 2012) or in the modelling of the reduced occupancy of heavy atoms (Schiltz et $a l ., 2004)$. However, there are often limitations to the use of such approaches on a systematic basis and, unless this is intentional, it is advisable to minimize radiation damage as much as possible. Here, we briefly describe data collection strategies aimed at reducing both systematic errors and radiation damage during MAD/SAD experiments and describe how such strategies can best be put into practice at the ESRF Structural Biology Beamlines.

\section{ESRF MAD Structural Biology Beamlines}

The ESRF operates three tunable-wavelength endstations each covering the energy range 6-20 keV: ID23-1 (Nurizzo et al., 2006), ID29 (de Sanctis et al., 2012) and ID30B (MuellerDieckmann et al., 2015). The three stations are equipped with similar diffractometers, MD2 (a microdiffractometer; Arinax, Moirans, France; Perrakis et al., 1999), MD2M (a minidiffractometer; Arinax, Moirans, France) and MD2S (a microdiffractometer for screening; Arinax, Moirans, France),

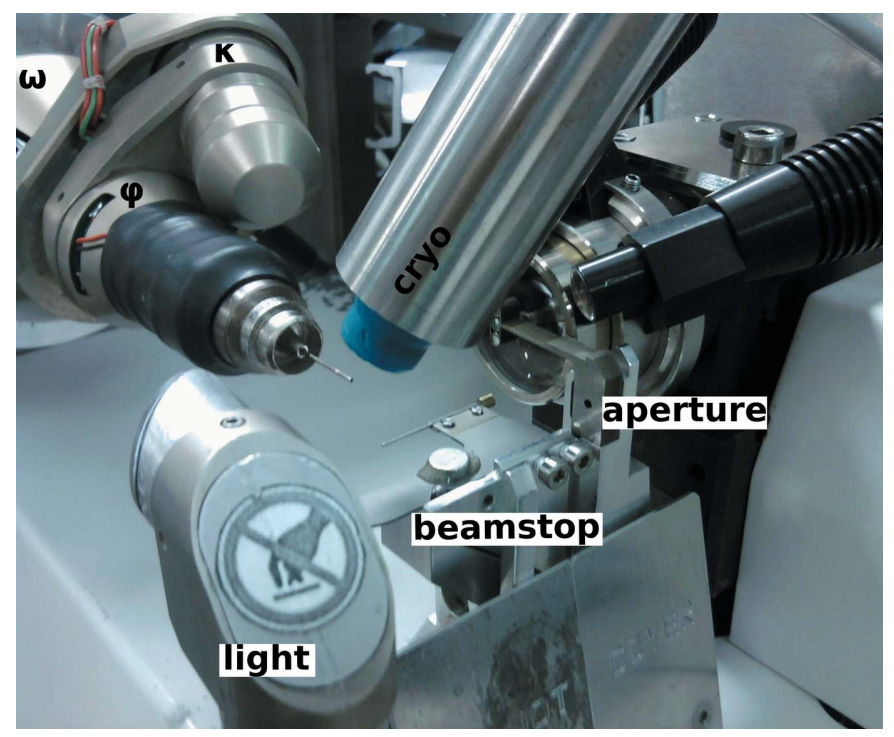

Figure 1

Close-up view of the sample environment on ID29 at the ESRF. Goniometer motors and main components are labelled. all with on-axis sample visualization. ID23-1 and ID29 have a fixed focal spot size at the sample position and are equipped with beam-defining apertures which allow tailoring of the X-ray beam size to a minimum dimension of $10 \mu \mathrm{m}$ in diameter (Fig. 1). ID30B offers a variable beamsize at the sample position ranging from 20 to $200 \mu \mathrm{m}$. All three endstations share a similar data-collection geometry $(\omega$ axis for oscillation scans horizontal and perpendicular to the X-ray beam) and are equipped with MK3 minikappa goniometers (Brockhauser et al., 2013) for crystal realignment and

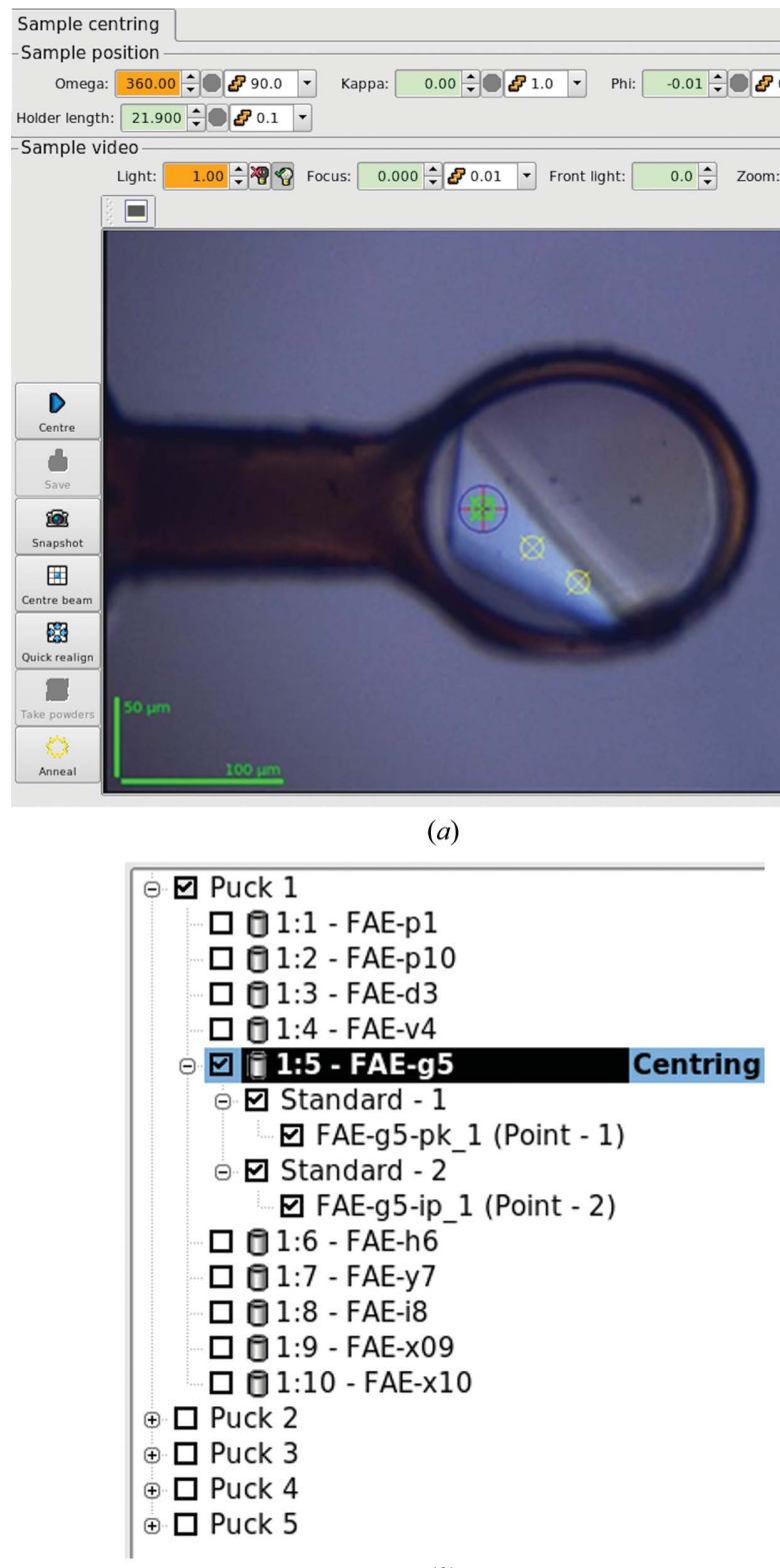

(b)

Figure 2

(a) Detail of the $M X C U B E 2$ interface. The active centred position is marked in lime and other saved positions are marked in yellow. To activate a saved position it is sufficient to click on it and multiple positions can be simultaneously activated. (b) Detail of the queue of $M X C U B E 2$. In the example shown a two-wavelength MAD (peak and inflection) data set is to be collected, with each data set collected from a different saved position of the sample 1:5 FAE-g5. 
fluorescence detectors for the measurement of absorptionedge scans, which are carried out at the click of a button and are automatically analysed with $\mathrm{CHOOCH}$ (Evans \& Pettifer, 2001) for the determination of anomalous scattering factors around the absorption edge end and of 'peak' and 'inflectionpoint' energies.

\section{The MXCUBE2 concept}

User control of experiments on ID23-1, ID29 and ID30B is, as for all of the ESRF facilities for MX, via the MXCuBE2 (http://www.esrf.eu/mxcube2 and https://github.com/mxcube) graphical user interface (GUI), the full functionality of which will be presented elsewhere (Oscarsson et al., in preparation). Briefly, $M X C U B E 2$, the successor to $M X C u B E$ (Gabadinho $e t$ al., 2010), has been designed to simplify the planning and execution of MX experiments via a single, integrated and intuitive interface, which facilitates the carrying out of experiments with elaborate data-collection strategies by minimizing error-prone iterative manual intervention. Two main aspects of $M X C U B E 2$ are relevant for experiments exploiting anomalous scattering in de novo crystal structure determination: firstly, $M X C u B E 2$ relies on the concept of 'saved positions' for data collection from any given sample (Fig. 2). Each saved position can be assigned a series of data collections to build up any complex data-collection scheme. Secondly, the $M X C U B E 2$ data-collection queue can be prefilled by expert experimental descriptors, such as EDNA (Incardona et al., 2009) and workflows (Brockhauser et al., 2012) that automate data collection and analysis.

\section{Good data-collection practice as implemented in MXCUBE2}

\subsection{Calculation of data-collection strategies}

Calculation of a suitable data-collection strategy is among the 'good practices' handed down by crystallographers (Dauter, 1999; Flot et al., 2006). Typically, the goal is to determine the minimal angular range and a convenient oscillation range (slicing) and to ensure that complete data with the desired multiplicity are collected. To ensure the calculation of optimal data-collection strategies at ESRF MX beamlines, the EDNA pipeline (Incardona et al., 2009) is used via the $M X C u B E 2$ 'Characterization' tab. The successor to $D N A$ (Leslie et al., 2002), EDNA has been developed to take advantage of the evolution of software such as LABELIT (Sauter et al., 2004), BEST (Bourenkov \& Popov, 2010) and RADDOSE (Paithankar \& Garman, 2010) to produce data collection strategies which take into account global radiation damage. Crystal symmetry is determined from two or four diffraction images using either LABELIT or MOSFLM; X-ray dose is calculated in RADDOSE, assuming an 'average' crystal composition, using the energy of the incident beam, photon flux and beam size, which are automatically provided by $M X C u B E 2$, and the dimensions of the crystal under study (Fig. 3). The dose is subsequently used in BEST to propose a
Table 1

Data-collection and processing statistics for data sets collected from a random orientation and with $c^{*}$ aligned with the rotation axis using the MK3 device.

Data were cut at $3.5 \AA$ resolution to properly compare the anomalous signal in the two data sets. No indication of global radiation damage, such as an increase in the unit-cell volume or in the overall $B$ factor, is observed in the data. Values in parentheses are for the outer shell.

\begin{tabular}{|c|c|c|}
\hline & Random & $\kappa\left(c^{*}\right)$ \\
\hline Beamline 。 & ID29 & ID29 \\
\hline Wavelength $(\AA)$ & 0.9791 & 0.9791 \\
\hline Temperature $(\mathrm{K})$ & 100 & 100 \\
\hline Detector & Pilatus3 6M & Pilatus3 6M \\
\hline Data-collection time (s) & 50 & 44 \\
\hline Photon flux (photons s ${ }^{-1}$ ) & $3.5 \times 10^{11}$ & $4.5 \times 10^{11}$ \\
\hline Crystal-to-detector distance (mm) & 429.2 & 463.1 \\
\hline Rotation range per image $\left(^{\circ}\right)$ & 0.05 & 0.1 \\
\hline Total rotation range $\left({ }^{\circ}\right)$ & 80 & 80 \\
\hline Space group & $P 4_{1} 2_{2} 2$ & $P 4_{1} 2{ }_{1} 2$ \\
\hline Unit-cell parameters $(\AA)$ & $\begin{array}{c}a=b=111.38 \\
c=65.40\end{array}$ & $\begin{array}{c}a=b=111.58 \\
c=65.59\end{array}$ \\
\hline Mosaicity $\left(^{\circ}\right)$ & 0.060 & 0.064 \\
\hline Resolution range $(\AA)$ & $40-3.5(3.83-3.50)$ & $40-3.5(3.83-3.50)$ \\
\hline Total No. of reflections & 30994 (7365) & $30586(7637)$ \\
\hline No. of unique reflections & $5505(1291)$ & $5500(1285)$ \\
\hline Completeness (\%) & $99.5(100)$ & $98.6(97.9)$ \\
\hline Multiplicity & $5.6(5.7)$ & $5.4(5.5)$ \\
\hline$\langle I / \sigma(I)\rangle$ & $36.8(26.1)$ & $49.4(38.4)$ \\
\hline$R_{\text {r.i.m. }} \dagger$ & $0.040(0.064)$ & $0.032(0.039)$ \\
\hline ISa & 31.04 & 31.52 \\
\hline DelAnom & $0.771(0.448)$ & $0.852(0.804)$ \\
\hline $\begin{array}{l}\text { Mid-Slope of Anom. Normal } \\
\text { Probability }\end{array}$ & 2.564 & 3.936 \\
\hline
\end{tabular}

$\dagger R_{\mathrm{r} . \mathrm{im} .}=\sum_{h k l}\{1 /[N(h k l)-1]\}^{1 / 2} \sum_{i}\left|I_{i}(h k l)-\langle I(h k l)\rangle\right| / \sum_{h k l} \sum_{i} I_{i}(h k l)$.

diffraction plan, which is added, in the form of a new data collection, to the $M X C U B E 2$ queue (Fig. 2). Crystal size can be measured directly from the sample view using a dedicated tool that converts lengths measured in pixels to micrometres. Users can also specify their requirements for data collection, such as preferred angular range or desired multiplicity, request an 'anomalous strategy' (Bourenkov \& Popov, 2010; Fig. 3b) and, if necessary, modify the parameters of the proposed strategy. For experiments aimed at de novo structure solution, the collection of highly redundant data is often advisable, and this becomes even more relevant when weak anomalous signals are to be exploited (Dauter \& Adamiak, 2001; Cianci et al., 2008; Akey et al., 2014; Weinert et al., 2015; Liu \& Hendrickson, 2015).

\subsection{Crystal reorientation}

Most MX beamlines at third-generation synchrotron sources are equipped with a single-axis goniometer to achieve high mechanical stability (Fig. 1), a requirement that has become particularly relevant since the advent of microfocus and microbeam beamlines (Flot et al., 2010; de Sanctis et al., 2012). However, when exploiting anomalous scattering in MX the use of a single-axis goniometer presents clear limitations for experiments designed to reduce systematic errors by either measuring the 'true multiplicity' (i.e. the multiplicity obtained by recording reflections from multiple different crystal 
orientations) or orienting a specific unit-cell axis parallel to the $\omega$ rotation axis so that Friedel mates can be measured on the same diffraction image. To overcome this limitation, ESRF tuneable beamlines are equipped with MK3 minikappa goniometers (Brockhauser et al., 2013) that allow crystal reorientation, while sample rotation is performed around $\omega$ (see Fig. 1 in Brockhauser et al., 2013). Truly redundant data can straightforwardly be obtained by combining data sets collected from the same crystal at different $\kappa$ angles. In this way, equivalent reflections are recorded on a different area of the two-dimensional detector and with different X-ray path lengths through the sample. $M X C u B E 2$ facilitates such experiments by allowing the creation of a queue of data collections, from the same or from different positions in a crystal, at different, user-defined $\kappa$ angles. A more complicated use of the functionality of the MK3 consists of reorienting the crystal so that an evenfold rotation axis of the unit-cell point group is oriented parallel to the $\omega$ rotation axis (Fig. 4). This allows the collection of both reflections in a Friedel pair on the same diffraction image, thus ensuring that they are measured at the same time and after the crystal has suffered the same X-ray dose. Calculating the $\kappa$ and $\varphi$ angles required for such a reorientation can be a complicated task that is beyond the scope of even experienced experimenters, as it requires knowledge of the beamline hardware configurations and limits. However, this procedure has been automated in the 'kappa reorientation' workflow (Brockhauser et al., 2012) directly interfaced with $M X C U B E 2$. In this workflow, two $1^{\circ}$ oscillation images collected $90^{\circ}$ apart in $\omega$ at $\kappa=0^{\circ}$ are used to identify the crystal Bravais lattice and calculate a crystal orientation matrix in the laboratory frame. Subsequently, the STAC server (Brockhauser et al., 2013) calculates the $\kappa$ and $\varphi$ angles that align an evenfold axis parallel to $\omega$. Once the crystal has been reoriented, the user centres the sample in the X-ray beam, a datacollection strategy for the optimum collection of anomalous data is then calculated with $E D N A$ and, upon the approval of the experimenter, executed. In Table 1 we report data-collection statistics from a crystal of the selenomethionine-derivative of the feruloyl esterase module of xylanase 10B from Clostridium thermocellum (Prates et al., 2001; 
PDB entry $1 \mathrm{gkk}$ ) obtained in space group $P 4_{2} 2{ }_{1} 2$. Two data sets were collected from different positions of a single crystal. The first data set was collected with the crystal in a random orientation, as harvested in the nylon loop, and the second with the $c^{*}$ axis aligned parallel to $\omega$. In both cases the datacollection strategies were as recommended by EDNA/BEST. As can be seen, reorientation along the $c^{*}$ axis results in a larger anomalous signal ('DelAnom' and 'Mid-Slope of Anom. Normal Probability' in Table 1), despite very similar total doses being used to collect data sets with very similar completeness and multiplicity.

\subsection{Inverse-beam data collection}

Although the MK3 is an extremely useful device for aligning crystals during MAD/SAD experiments (see above) or for aligning crystals such that a long unit axis is parallel to $\omega$, a combination of unfortunate crystal orientation inside the sample loop and the limited $\left(24^{\circ}\right) \alpha$ opening angle of the MK3 may mean that the reorientation required is out of reach. In such cases it may well be desirable in MAD/SAD experiments to ensure the collection of both reflections in a Friedel pair

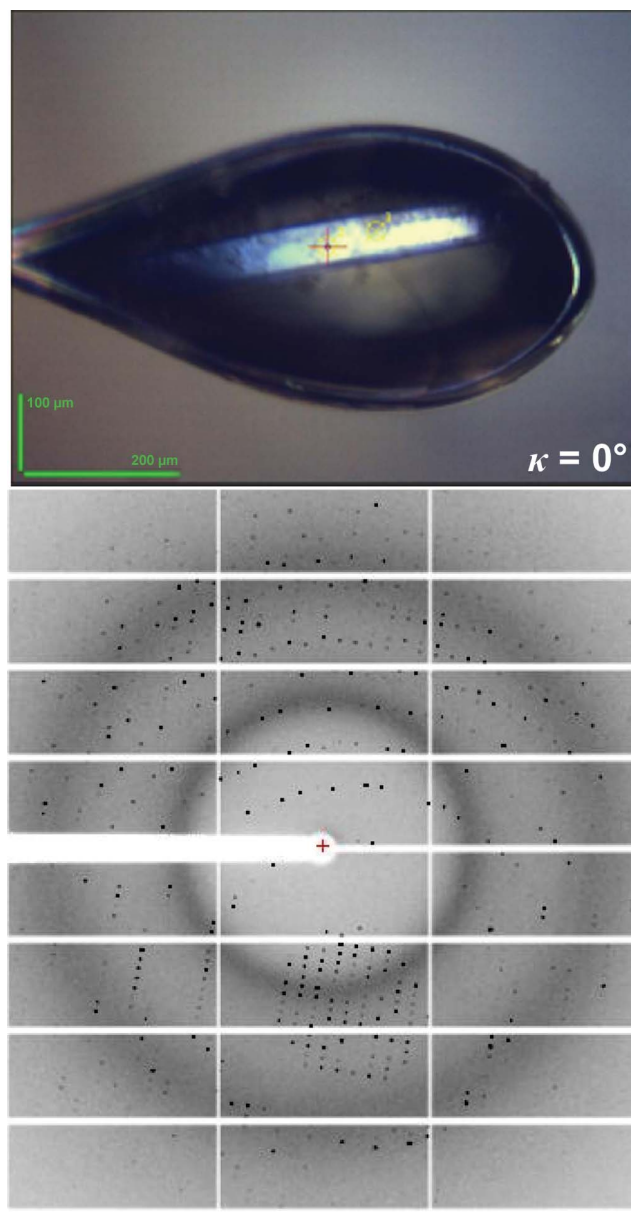

(a)
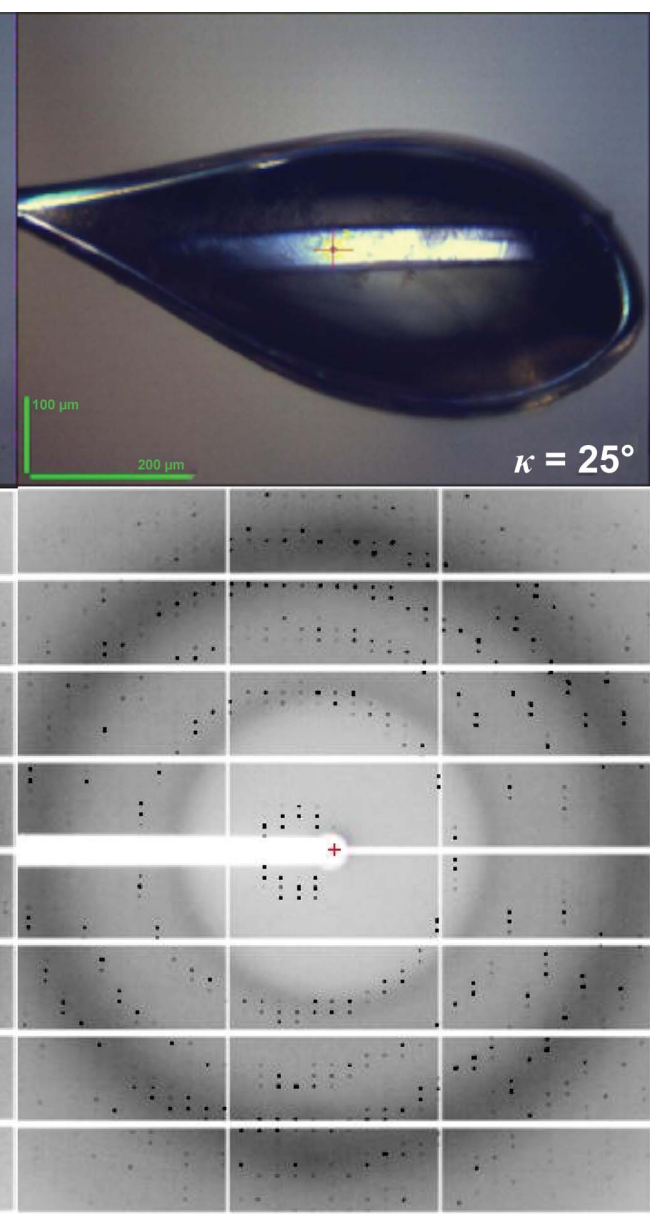

(b)
Figure 4

Effect of the alignment of a rod-like crystal along one of its evenfold symmetry axes, starting from $(a)$ a random orientation, the result of the harvesting from the crystallization drop, to $(b)$ a final reorientation with, in this case, $c^{*}$ parallel to $\omega$. close together in time and in X-ray dose by resorting to inverse-beam geometry (Hendrickson et al., 1985; Dauter, 1997; Fig. 5). In an inverse-beam experiment the two reflections in a Freidel pair are collected at rotation angles $\omega$ and $\omega$ $+180^{\circ}$, respectively, and an inverse-beam geometry experiment consists of subdividing the total angular range to be collected into two data sets $180^{\circ}$ apart in $\omega$ and collecting them, often broken down into 'subwedges', in sequence (Figs. $3 c$ and 5). The beamline-control software takes care of the correct starting angles, image and run numbering for each data set. The two data sets sets are processed separately and then scaled together to provide the final data set. Although, to our knowledge a systematic study on the beneficial use of inverse-beam geometry is not available, many examples of successful phasing experiments using this technique have been reported in the literature (Liu et al., 2012, 2013; Akey et al., 2014).

\subsection{Interleaved MAD data collection}

In the early days, MAD experiments were complicated and required intervention from the local staff to properly prepare the beamline (i.e. change the energy and realign the beamline optical components and sample environment) at each wavelength at which MAD data were collected. The degree of automation achieved during the last decade at the ESRF MX beamlines (Arzt et al., 2005) and at other synchrotrons worldwide (Soltis et al., 2008; Stepanov et al., 2011; Cork et al., 2006) has simplified and stabilized the operation of tunable MX beamlines to the extent that energy changes during MAD experiments are now almost transparent to the user. This increase in userfriendliness has allowed users to concentrate on the optimization of experiment design, which is particularly relevant when extracting small anomalous signals for de novo structure solution or when samples are sensitive to radiation damage. As already noted, $M X C U B E 2$ is designed to allow the modular assembly of data-collection protocols and gives the opportunity to build the experiment sequences. One such experiment sequence available as a workflow in $M X C U B E 2$ and in which subwedges of complete 
diffraction data sets are alternately collected at different energies is 'interleaved MAD' (Finke et al., 2016), a protocol which ensures that the dispersive differences between the same reflections in different data sets are minimally affected by X-ray damage. To demonstrate the ease of carrying out such a data-collection protocol, we collected, from two different positions of the same crystal of the feruloyl esterase module of xylanase 10B from $C$. thermocellum (Prates et al., 2001) obtained in space group $P 2_{1} 2_{1} 2_{1}$, a 'classic' two-wavelength MAD data set and a MAD data set in which the collection of data at the two wavelengths was interleaved by $10^{\circ}$ subwedges. The resulting data processing statistics are reported in Table 2 and show the individual data sets to be of similar quality. The interface for the 'interleaved MAD' workflow permits interleaving of the collection of MAD diffraction data at up to four different energies and, furthermore, can be used in combination with inverse-beam geometry (Fig. $3 d$ ). Evolution of the workflow to provide new interleaving protocols is straightforward and can easily be implemented. For example, interleaving of the collection of diffraction data at different detector distances or $\kappa$ angles, for example, which should reduce systematic detector errors or compensate for suboptimal absorption correction at lower energy, is accessible by just building the desired datacollection sequence in the MXCUBE2 GUI.

\subsection{Multi-positional data collection}

The advent of microfocus (Flot et al., 2010) and microbeam (de Sanctis et al., 2012) endstations dedicated to MX allows the measurement of diffraction data from smaller samples and maximizes signal-to-noise ratios by matching beam and crystal sizes or the scanning of a larger crystal to find its best diffracting area (Sanishvili et al., 2008; Bowler et al., 2010). Microbeams also allow the collection of diffraction data from different parts of crystals larger than the X-ray beam. $M X C U B E 2$ allows users to centre different positions of large crystals in the X-ray beam, to save and store these positions and to link each position with a series of data collections (Fig. 2). In this way, it is possible to sequentially collect multiple data sets from the same sample. This functionality enables different possible strategies: by collecting complete data sets at different positions it is possible to increase multiplicity while escaping radiation damage; for highly radiationsensitive samples partial data sets can be collected at each position and merged to produce a single complete data set to higher resolution than might otherwise have been the case; in MAD experiments data sets at different energies can be collected from different positions of the same crystal. This data-collection method is fully exploited in the MeshAndCollect data-collection workflow (Zander et al., 2015), in which the positions of micrometre-sized crystals are identified by diffraction and automatically saved and a partial data set is collected at each centred point. While MeshAndCollect completely automates the procedure, beamline users can optically select the centring positions and define a data collection plan for each of them. The same rationale can be applied when collecting data sets at different wavelengths to perform MAD/SAD phasing experiments. In fact, although SAD phasing has become extremely popular (also thanks to the improvement in phasing and density-modification software), the fast performance of pixel detectors and beamline automation nowadays allows a second data set to rapidly be collected at another energy to obtain experimental phases of much higher quality (González, 2003). In order to avoid

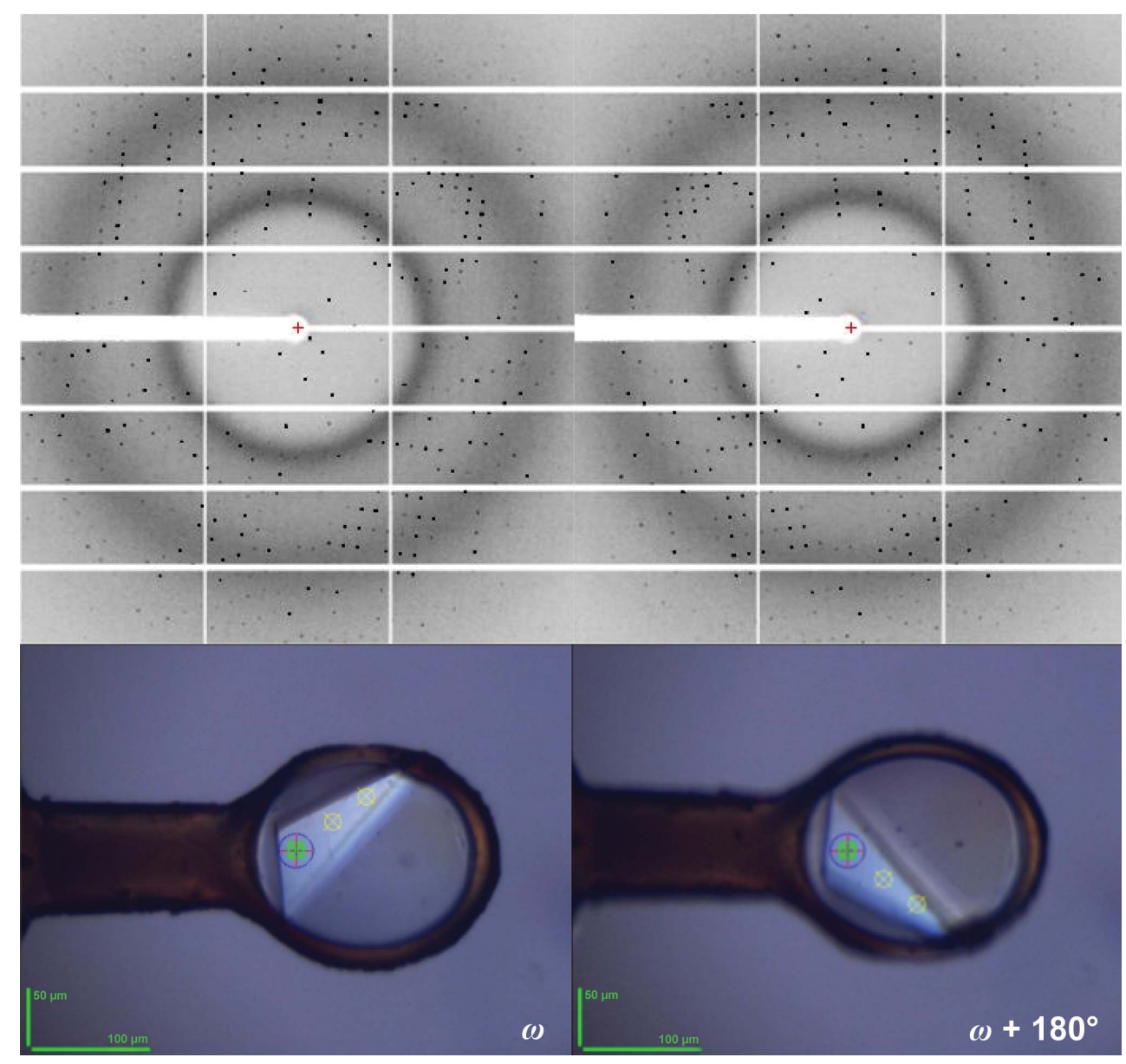

Figure 5

Inverse-beam data collection allows the measurement of reflections in a Friedel pair close together in time by alternately collecting diffraction images at $\omega$ (left) and $\omega+180^{\circ}$ (right). 
Table 2

Data-collection parameters and processing statistics for the feruloyl esterase module of xylanase 10B from C. thermocellum (Prates et al., 2001; PDB entry 1gkk).

Data sets were collected from a $P 2_{1} 2_{1} 2_{1}$ crystal in the form of a 'classic' two-wavelength MAD experiment and a 'interleaved' MAD experiment, in which data are collected in $10^{\circ}$ subwedges alternating between the two energies. Data were cut at $3.5 \AA$ resolution to properly compare the anomalous signal in the two data sets. No indication of global radiation damage, such as an increase in the unit-cell volume or in the overall $B$ factor, is observed in the collected data. Values in parentheses are for the outer shell.

\begin{tabular}{|c|c|c|c|c|}
\hline & Peak 'classic' & Inflection 'classic' & Peak 'interleaved' & Inflection 'interleaved' \\
\hline Beamline & ID29 & ID29 & ID29 & ID29 \\
\hline Wavelength (§) & 0.9791 & 0.9793 & 0.9791 & 0.9793 \\
\hline Temperature (K) & 100 & 100 & 100 & 100 \\
\hline Detector & Pilatus3 6M & Pilatus3 6M & Pilatus3 6M & Pilatus3 6M \\
\hline Data-collection time (s) & 24 & 24 & 24 & 24 \\
\hline Photon flux (photons s ${ }^{-1}$ ) & $1.5 \times 10^{11}$ & $1.5 \times 10^{11}$ & $1.5 \times 10^{11}$ & $1.5 \times 10^{11}$ \\
\hline Crystal-to-detector distance ( $\mathrm{mm})$ & 391.77 & 391.75 & 391.73 & 391.56 \\
\hline Rotation range per image $\left({ }^{\circ}\right)$ & 0.1 & 0.1 & 0.1 & 0.1 \\
\hline Total rotation range $\left({ }^{\circ}\right)$ & 120 & 120 & 120 & 120 \\
\hline Space group & $P 2_{1} 2_{1} 2_{1}$ & $P 2_{1} 2_{1} 2_{1}$ & $P 2_{1} 2_{1} 2_{1}$ & $P 2_{1} 2_{1} 2_{1}$ \\
\hline Unit-cell parameters $(\AA)$ & $\begin{array}{l}a=64.82, b=108.42 \\
\quad c=113.21\end{array}$ & $\begin{array}{l}a=64.86, b=108.50 \\
\quad c=113.29\end{array}$ & $\begin{array}{l}a=64.80, b=108.42, \\
\quad c=113.16\end{array}$ & $\begin{array}{l}a=64.80, b=108.40 \\
\quad c=113.17\end{array}$ \\
\hline Mosaicity $\left({ }^{\circ}\right)$ & 0.039 & 0.040 & 0.042 & 0.043 \\
\hline Resolution range $(\AA)$ & $40-3.5(3.83-3.50)$ & $40-3.5(3.83-3.50)$ & $40-3.5(3.83-3.50)$ & $40-3.5(3.83-3.50)$ \\
\hline Total No. of reflections & $44499(10699)$ & $44430(10671)$ & $44492(10384)$ & $44418(10620)$ \\
\hline No. of unique reflections & $10397(2478)$ & $10367(2472)$ & $10384(2465)$ & $10359(2470)$ \\
\hline Completeness (\%) & $98.9(99.5)$ & $98.7(99.3)$ & $98.9(99.3)$ & $98.8(99.5)$ \\
\hline Multiplicity & $4.3(4.3)$ & $4.3(4.3)$ & $4.3(4.3)$ & $4.3(4.3)$ \\
\hline$\langle I / \sigma(I)\rangle$ & $28.5(27.1)$ & $28.8(26.9)$ & $31.1(28.9)$ & $30.2(27.9)$ \\
\hline$R_{\text {r.i.m. }} \dagger$ & $0.046(0.044)$ & $0.045(0.044)$ & $0.042(0.042)$ & $0.043(0.043)$ \\
\hline ISa & 21.82 & 22.50 & 25.62 & 25.06 \\
\hline DelAnom & $0.679(0.678)$ & $0.696(0.674)$ & $0.730(0.679)$ & $0.718(0.661)$ \\
\hline Mid-Slope of Anom. Normal Probability & 2.610 & 2.515 & 2.733 & 2.693 \\
\hline
\end{tabular}

$\dagger R_{\text {r.i.m. }}=\sum_{h k l}\{1 /[N(h k l)-1]\}^{1 / 2} \sum_{i}\left|I_{i}(h k l)-\langle I(h k l)\rangle\right| / \sum_{h k l} \sum_{i} I_{i}(h k l)$.

contamination with radiation damage in the second (or a third or subsequent) data set, it would be advisable to collect each data set from an undamaged volume (Fig. $2 b$ ). A caveat when applying such strategies is that the diffraction quality over the length and/or area of a crystal can often be heterogeneous (Sanishvili et al., 2008; Bowler et al., 2010); the recommendation would then be to characterize each position (using $E D N A / B E S T)$ to make sure that the crystal quality is uniform. For the reason above, although the execution of helical (Flot $e t$ $a l ., 2010$ ) or vector (Pothineni et al., 2014) data collections is routinely available in $M X C u B E 2$, these must be carefully pondered before they are carried out.

\section{Conclusions}

Experimental phasing by anomalous dispersion techniques are the most successful and common ab initio methods for structure determination. The success of an anomalous dispersion experiment strongly relies on the data quality, and this becomes more significant when small anomalous signals are expected. In such circumstances, experiment design and the tools to perform experiments play a major role. Here, we have presented a list of the most common 'best practices' to be used in the collection of high-quality diffraction data for use in MAD or SAD experiments. $M X C U B E 2$, the experimentcontrol GUI available at the ESRF Structural Biology Beamlines, facilitates these by a number of different means. $M X C U B E 2$ allows the use of EDNA, RADDOSE and BEST to propose data-collection strategies that take into account crystal decay caused by radiation damage, it allows the straightforward implementation of complex data-collection protocols such as inverse-beam and interleaved data-collection experiments and it allows the efficient use of minikappa goniometers and of multi-position and multi-crystal data collections. Moreover, the $M X C U B E 2$ data-collection queue modularity smooths the way for the integration of novel datacollection methods and data analysis, as already demonstrated with the MeshAndCollect pipeline. $M X C U B E 2$ is constantly evolving to incorporate new experiment types and to hand them over to the MX user community.

\section{Acknowledgements}

The continuous development of the Structural Biology Beamlines has been possible thanks to the support from the ESRF Beamline Control Unit, all Structural Biology Group members and the feedback from our user community. MXCuBE2 is the fruit of the collaboration of the partners of the MXCuBE consortium, which includes ESRF, EMBL, the MAX IV Laboratory, Synchrotron SOLEIL, HZB-BESSY, DESY, the ALBA Synchrotron and Global Phasing Ltd.

\section{References}

Akey, D. L., Brown, W. C., Konwerski, J. R., Ogata, C. M. \& Smith, J. L. (2014). Acta Cryst. D70, 2719-2729.

Arzt, S. et al. (2005). Prog. Biophys. Mol. Biol. 89, 124-152.

Berglund, G. I., Carlsson, G. H., Smith, A. T., Szöke, H., Henriksen, A. \& Hajdu, J. (2002). Nature (London), 417, 463-468. 
Berman, H., Henrick, K., Nakamura, H. \& Markley, J. L. (2007). Nucleic Acids Res. 35, D301-D303.

Bourenkov, G. P. \& Popov, A. N. (2010). Acta Cryst. D66, 409-419.

Bowler, M. W., Guijarro, M., Petitdemange, S., Baker, I., Svensson, O., Burghammer, M., Mueller-Dieckmann, C., Gordon, E. J., Flot, D., McSweeney, S. M. \& Leonard, G. A. (2010). Acta Cryst. D66, 855-864.

Brockhauser, S., Ravelli, R. B. G. \& McCarthy, A. A. (2013). Acta Cryst. D69, 1241-1251.

Brockhauser, S., Svensson, O., Bowler, M. W., Nanao, M., Gordon, E., Leal, R. M. F., Popov, A., Gerring, M., McCarthy, A. A. \& Gotz, A. (2012). Acta Cryst. D68, 975-984.

Cianci, M., Helliwell, J. R. \& Suzuki, A. (2008). Acta Cryst. D64, 1196-1209.

Cork, C., O’Neill, J., Taylor, J. \& Earnest, T. (2006). Acta Cryst. D62, 852-858.

Dauter, Z. (1997). Methods Enzymol. 276, 326-344.

Dauter, Z. (1999). Acta Cryst. D55, 1703-1717.

Dauter, Z. (2002). Curr. Opin. Struct. Biol. 12, 674-678.

Dauter, Z. \& Adamiak, D. A. (2001). Acta Cryst. D57, 990-995.

Dauter, Z., Dauter, M., de La Fortelle, E., Bricogne, G. \& Sheldrick, G. M. (1999). J. Mol. Biol. 289, 83-92.

Dauter, Z., Dauter, M. \& Dodson, E. J. (2002). Acta Cryst. D58, 494-506.

DiMaio, F., Terwilliger, T. C., Read, R. J., Wlodawer, A., Oberdorfer, G., Wagner, U., Valkov, E., Alon, A., Fass, D., Axelrod, H. L., Das, D., Vorobiev, S. M., Iwaï, H., Pokkuluri, P. R. \& Baker, D. (2011). Nature (London), 473, 540-543.

Ennifar, E., Carpentier, P., Ferrer, J.-L., Walter, P. \& Dumas, P. (2002). Acta Cryst. D58, 1262-1268.

Evans, G. \& Pettifer, R. F. (2001). J. Appl. Cryst. 34, 82-86.

Evans, G., Polentarutti, M., Djinovic Carugo, K. \& Bricogne, G. (2003). Acta Cryst. D59, 1429-1434.

Finke, A. D., Panepucci, E., Vonrhein, C., Wang, M., Bricogne, G. \& Oliéric, V. (2016). Methods Mol. Biol. 1320, 175-191.

Flot, D., Gordon, E. J., Hall, D. R., Leonard, G. A., McCarthy, A., McCarthy, J., McSweeney, S., Mitchell, E., Nurizzo, D., Ravelli, R. G. B. \& Shepard, W. (2006). Acta Cryst. D62, 65-71.

Flot, D., Mairs, T., Giraud, T., Guijarro, M., Lesourd, M., Rey, V., van Brussel, D., Morawe, C., Borel, C., Hignette, O., Chavanne, J., Nurizzo, D., McSweeney, S. \& Mitchell, E. (2010). J. Synchrotron Rad. 17, 107-118.

Gabadinho, J. et al. (2010). J. Synchrotron Rad. 17, 700-707.

González, A. (2003). Acta Cryst. D59, 1935-1942.

Hendrickson, W. A. (1991). Science, 254, 51-58.

Hendrickson, W. A., Smith, J. L. \& Sheriff, S. (1985). Methods Enzymol. 115, 41-55.

Hendrickson, W. A. \& Teeter, M. M. (1981). Nature (London), 290, 107-113.

Holton, J. M. (2009). J. Synchrotron Rad. 16, 133-142.

Incardona, M.-F., Bourenkov, G. P., Levik, K., Pieritz, R. A., Popov, A. N. \& Svensson, O. (2009). J. Synchrotron Rad. 16, 872-879.

Leiros, H.-K. S., McSweeney, S. M. \& Smalås, A. O. (2001). Acta Cryst. D57, 488-497.

Leslie, A. G. W., Powell, H. R., Winter, G., Svensson, O., Spruce, D., McSweeney, S., Love, D., Kinder, S., Duke, E. \& Nave, C. (2002). Acta Cryst. D58, 1924-1928.

Liu, Q., Dahmane, T., Zhang, Z., Assur, Z., Brasch, J., Shapiro, L., Mancia, F. \& Hendrickson, W. A. (2012). Science, 336, 1033-1037.
Liu, Q. \& Hendrickson, W. A. (2015). Curr. Opin. Struct. Biol. 34, 99-107.

Liu, Q., Liu, Q. \& Hendrickson, W. A. (2013). Acta Cryst. D69, 13141332.

McCoy, A. J., Grosse-Kunstleve, R. W., Adams, P. D., Winn, M. D., Storoni, L. C. \& Read, R. J. (2007). J. Appl. Cryst. 40, 658-674.

Mueller-Dieckmann, C., Bowler, M. W., Carpentier, P., Flot, D., McCarthy, A. A., Nanao, M. H., Nurizzo, D., Pernot, P., Popov, A., Round, A., Royant, A., de Sanctis, D., von Stetten, D. \& Leonard, G. A. (2015). Eur. Phys. J. Plus, 130, 70.

Nurizzo, D., Mairs, T., Guijarro, M., Rey, V., Meyer, J., Fajardo, P., Chavanne, J., Biasci, J.-C., McSweeney, S. \& Mitchell, E. (2006). J. Synchrotron Rad. 13, 227-238.

Owen, R. L., Rudiño-Piñera, E. \& Garman, E. F. (2006). Proc. Natl Acad. Sci. USA, 103, 4912-4917.

Paithankar, K. S. \& Garman, E. F. (2010). Acta Cryst. D66, 381-388.

Perrakis, A., Cipriani, F., Castagna, J.-C., Claustre, L., Burghammer, M., Riekel, C. \& Cusack, S. (1999). Acta Cryst. D55, 1765-1770.

Pothineni, S. B., Venugopalan, N., Ogata, C. M., Hilgart, M. C., Stepanov, S., Sanishvili, R., Becker, M., Winter, G., Sauter, N. K., Smith, J. L. \& Fischetti, R. F. (2014). J. Appl. Cryst. 47, 19921999.

Prates, J. A. M., Tarbouriech, N., Charnock, S. J., Fontes, C. M. G. A., Ferreira, L. M. A. \& Davies, G. J. (2001). Structure, 9, 1183-1190.

Ramagopal, U. A., Dauter, Z., Thirumuruhan, R., Fedorov, E. \& Almo, S. C. (2005). Acta Cryst. D61, 1289-1298.

Ravelli, R. B. G., Leiros, H.-K. S., Pan, B., Caffrey, M. \& McSweeney, S. (2003). Structure, 11, 217-224.

Ravelli, R. B. G., Nanao, M. H., Lovering, A., White, S. \& McSweeney, S. (2005). J. Synchrotron Rad. 12, 276-284.

Read, R. J. (2001). Acta Cryst. D57, 1373-1382.

Rice, L. M., Earnest, T. N. \& Brunger, A. T. (2000). Acta Cryst. D56, 1413-1420.

Sanctis, D. de et al. (2012). J. Synchrotron Rad. 19, 455-461.

Sanctis, D. de \& Nanao, M. H. (2012). Acta Cryst. D68, 1152-1162.

Sanishvili, R., Nagarajan, V., Yoder, D., Becker, M., Xu, S., Corcoran, S., Akey, D. L., Smith, J. L. \& Fischetti, R. F. (2008). Acta Cryst. D64, 425-435.

Sauter, N. K., Grosse-Kunstleve, R. W. \& Adams, P. D. (2004). J. Appl. Cryst. 37, 399-409.

Schiltz, M., Dumas, P., Ennifar, E., Flensburg, C., Paciorek, W., Vonrhein, C. \& Bricogne, G. (2004). Acta Cryst. D60, 1024-1031.

Schlichting, I., Berendzen, J., Chu, K., Stock, A. M., Maves, S. A., Benson, D. E., Sweet, R. M., Ringe, D., Petsko, G. A. \& Sligar, S. G. (2000). Science, 287, 1615-1622.

Seltzer, S. M. (1993). Radiat. Res. 136, 147-170.

Smith, J. L. (1991). Curr. Opin. Struct. Biol. 1, 1002-1011.

Soltis, S. M. et al. (2008). Acta Cryst. D64, 1210-1221.

Stepanov, S., Makarov, O., Hilgart, M., Pothineni, S. B., Urakhchin, A., Devarapalli, S., Yoder, D., Becker, M., Ogata, C., Sanishvili, R., Venugopalan, N., Smith, J. L. \& Fischetti, R. F. (2011). Acta Cryst. D67, 176-188.

Weik, M., Ravelli, R. B. G., Kryger, G., McSweeney, S., Raves, M. L., Harel, M., Gros, P., Silman, I., Kroon, J. \& Sussman, J. L. (2000). Proc. Natl Acad. Sci. USA, 97, 623-628.

Weinert, T. et al. (2015). Nature Methods, 12, 131-133.

Zander, U., Bourenkov, G., Popov, A. N., de Sanctis, D., Svensson, O., McCarthy, A. A., Round, E., Gordeliy, V., Mueller-Dieckmann, C. \& Leonard, G. A. (2015). Acta Cryst. D71, 2328-2343. 